\title{
Some Theorems upon Negative Energy Density of a Quantum Free Scalar Field in an Inertial World Line of the Minkowski Space-Time
}

\author{
M.A. Grado-Caffaro and M. Grado-Caffaro \\ M.A. Grado-Caffaro and M. Grado-Caffaro- Scientific Consultants, C/ Julio Palacios 11, 9-B, 28029-Madrid \\ (Spain) \\ E-mail: ma.grado-caffaro@sapienzastudies.com
}

\begin{abstract}
After presenting a lemma, two theorems on negative energy density associated with a quantum free scalar field are established. The first theorem provides a lower bound for a non-negative weight function whose existence is guaranteed by the lemma. The above energy density is evaluated over an inertial world line of the Minkowski space-time. The second theorem provides an upper bound for the averaged (with respect to the sampling function) absolute expectation value of the negative energy-density function. In particular, a complex-valued sampling function is introduced by the first time so a more generalized formulation is proposed.
\end{abstract}

Keywords: Negative energy density, quantum field, inertial world line, Minkowski space-time.

Mathematics Subject Classification: 81T10, 81T20, 81T55, 81T70.

PACS numbers: 03.70.+k; 04.62.+v; 98.80.Qc

\section{Introduction}

In quantum field theory, one can find very interesting examples on negative energy density (see, for instance, ref.[1]). Referring to the general context of quantum physics, there are cases in which energy density can be negative. In this respect, the Casimir effect (see, for example, ref.[1]) is certainly relevant: the quantum fluctuations of the electromagnetic field in the vacuum give rise to a macroscopic effect (Casimir effect) by which the electromagnetic-energy density is negative in the cavity formed by two conducting plates separated by a certain distance. One can think that, in principle, the energy density associated with a quantum field can be made arbitrarily negative at any point of the space-time by choosing a suitable state. Despite considering a classical field in which energy densities are positive in every point of the space-time, the renormalized energy density of the field quantized from the above classical field can be zero and can take either strictly positive or strictly negative values (note that the above density must be continuous). In principle, the renormalized energy density in question may even be made arbitrarily negative at a given point of the space-time. As a matter of fact, arbitrarily negative expectation values of the energy density can be measured after quantization even though the spatially integrated density holds non-negative.

The aim of the present paper lies on proving two useful theorems about negative energy density of a free scalar field on an inertial world line of the Minkowski space-time. In this context, we may consider, for example, Dirac or Klein-Gordon fields. On the other hand, a key ingredient in the hypotheses of the above theorems is assuming realistically that the energy density of the aforementioned free scalar field cannot take on negative values arbitrarily. Really, negative energy density is constrained to certain conditions so that the negativeness of the density is not arbitrary; those conditions have been formulated currently by means of quantum inequalities. Before establishing the theorems in question, we will enunciate a lemma which certainly contributes enough to the clarification of the state of the art. In addition, a complex-valued sampling function will be introduced. 


\section{Theory}

Our starting point is the following lemma. We enunciate:

Lemma. Given a quantum free scalar field, we consider the energy density of the field measured along an inertial world line of the Minkowski space-time. Let $\langle\rho(t)\rangle$ be the expectation value of the energy density $\rho(\vec{r}, t)$ of the field in question along the above inertial line. Then, for every complex-valued time-dependent function $f(t)$ (the so-called sampling function) and for all physically admissible states, there exists a non-negative real-valued function (denoted by $p(u)$ ) of polynomial growth such that:

$$
\int_{0}^{\infty}|f(t)|^{2}\langle\rho(t)\rangle d t \geq-\int_{0}^{\infty} p(u)|\tilde{f}(u)|^{2} d u
$$

where $\tilde{f}(u)$ is the Fourier transform of $f(t)$ so $\tilde{f}(u)$ can be defined over the angular- frequency domain. On the other hand, the integral on the right-hand side of formula (1) is assumed to be convergent. This implies that the integral on the left-hand side of (1) is absolutely convergent because, multiplying by -1 both sides of relationship (1), we have:

$$
\left.\int_{0}^{\infty}|f(t)|^{2}\left|\langle\rho(t)\rangle d t \leq \int_{0}^{\infty} p(u)\right| \tilde{f}(u)\right|^{2} d u
$$

that is:

$$
(f,|\langle\rho\rangle| f)_{t} \leq(\tilde{f}, p \tilde{f})_{u}
$$

Where, denotes the standard scalar product in a Hilbert space, $|\langle\rho(t)\rangle|$ and $p(u)$ being the weight functions of the above product in the time and angular-frequency domains, respectively. At this point, notice the non-negativeness of these weight functions. In refs.[2-4], smoothness of $f(t)$ (which was supposed as a real-valued function) was assumed but, in reality, this condition can be removed. In addition, in refs.[2-4], it was assumed that the support of $f(t)$ is compact; however, this condition is unnecessary. By the way, refs.[2-4] are an unfortunate example of wrong work.

Now we enunciate the following:

Theorem 1. Assume that the expectation value of the energy density satisfies the relation namely $\langle\rho(t)\rangle \equiv-\varepsilon$ with $\varepsilon>0$. Then, the function $p(u)$ is bounded below if and only if $p(u) \geq \varepsilon /(2 \pi), \forall u \in[0, \infty]$.

Proof. From the hypothesis, we infer that the left-hand side of expression (2) verifies:

$$
\int_{0}^{\infty}|f(t)|^{2}|\langle\rho(t)\rangle| d t=\varepsilon \int_{0}^{\infty}|f(t)|^{2} d t
$$

It is clear that from the conjunction of formulae (2) and (3), one gets:

$$
\varepsilon \int_{0}^{\infty}|f(t)|^{2} d t \leq \int_{0}^{\infty} p(u)|\tilde{f}(u)|^{2} d u
$$

Now we apply the Parseval's theorem namely:

$$
\int_{0}^{\infty}|f(t)|^{2} d t=\frac{1}{2 \pi} \int_{0}^{\infty}|\tilde{f}(u)|^{2} d u
$$

By combining (4) with (5), it follows: 


$$
\int_{0}^{\infty}\left[\frac{2 \pi}{\varepsilon} p(u)-1\right]|\tilde{f}(u)|^{2} d u \geq 0
$$

In order for inequality (6) to be satisfied, it is necessary and sufficient that the first factor of the integrand be non-negative, i.e., if and only if $p(u) \geq \varepsilon /(2 \pi), \forall u \in[0, \infty]$, so theorem 1 is proved.

Corollary. Since $p(u) \geq \varepsilon /(2 \pi)$, we deduce that $\int_{0}^{\infty} p(u) d u=\infty$.

Remark. From the hypothesis of theorem 1, one has that $\int_{0}^{\infty}|\langle\rho(t)\rangle| d t=\infty$.

Now we state:

Theorem 2. Assume that there exists a function $\hat{F}^{-1} p(u)$ for $t \geq 0$ where $\hat{F}$ is the Fourier-transform operator which, for every admissible function $\phi(t)$ defined at $t \geq 0$, leads to that $\hat{F} \phi(t) \equiv \tilde{\phi}(u) \equiv \int_{0}^{\infty} \phi(t) \exp (-i u t) d t$. Then, it is satisfied that:

$$
\langle\mid\langle\rho\rangle\rangle_{f} \leq 2 \pi\left\langle\hat{F}^{-1} p\right\rangle_{\zeta}
$$

where $\zeta \equiv \zeta(t, u)=e^{-i u t} \bar{f}(t) * f(t)$, the overbar denoting complex conjugate and the asterisk standing for convolution.

Proof. The integrand on the right-hand side of formula (2) can be expressed as $\hat{F}\left(\bar{f}(t) * f(t) * \hat{F}^{-1} p(u)\right)$. This fact, in conjunction with relationship (2) and with $p_{\min }=\varepsilon /(2 \pi)$ (theorem 1), leads straightforwardly to inequality (7).

Corollary. The double integral on the left-hand side of (7) is minorized, after Parseval's theorem, by $p_{\min } \int_{0}^{\infty}|\tilde{f}(u)|^{2} d u$.

\section{Conclusions}

We have proved two interesting theorems after clarifying a lemma which has been the starting point of our formulation. Certainly, the fact that the expectation value of the energy density associated with a quantum free scalar field measured on an inertial world line of the Minkowski space-time may be negative becomes relevant since this fact has important implications on a number of aspects of quantum physics, aspects related to quantum field theory being perhaps the most significant ones [5-15]. By theorem 1, we have deduced the minimal value taken on by $p(u)$; this is a useful condition to be fulfilled by the function $p(u)$ whose relevance is clearly manifest. On the other hand, theorem 2 provides an inequality (formula (7)) involving the aforementioned minimal value, $f(t)$, and the inverse Fourier transform of $p(u)$. At this point, we recall that we have introduced a restriction on $p(u)$ namely: there exists $\hat{F}^{-1} p(u)$ which, in practice, really exists. Finally, one may think upon further studies concerning non-inertial world lines of the Minkowski space-time.

\section{References}

1. M. Bordag, U. Mohideen, V.M. Mostepanenko, Phys. Rep. 353 (2001) 1.

2. C.J. Fewster, S.P. Eveson, Phys. Rev. D 58 (1998) 084010.

3. C.J. Fewster, E. Teo, Phys. Rev. D 59 (1999) 104016. 
4. S.P. Eveson, Ch.J. Fewster, R. Verch, Ann. Henri Poincaré 6 (2005) 1-30.

5. M.A. Grado-Caffaro, M. Grado-Caffaro, Nonlin. Opt. Quant. Opt. 42 (2011) 103-108.

6. M.A. Grado-Caffaro, M. Grado-Caffaro, Int. J. Theor. Phys. Group Theory Nonlin. Opt. 17 (2013) 91-100.

7. M.A. Grado-Caffaro, M. Grado-Caffaro, Adv. Studies Theor. Phys. 7 (2013) 549-554.

8. M.A. Grado-Caffaro, M. Grado-Caffaro, Optik 122 (2011) 1216-1217.

9. M.A. Grado-Caffaro, M. Grado-Caffaro, Optik 124 (2013) 4095-4096.

10.M.S. Morris, K.S. Thorne, U. Yurtsever, Phys. Rev. Lett. 61 (1988) 1446.

11.M.S. Morris, K.S. Thorne, Amer. J. Phys. 56 (1988) 395.

12.H. Bostelmann, D. Cadamuro, Phys. Rev. D 93 (2016) 065001.

13.L.M. Butcher, Phys. Rev. D 90 (2014) 024019.

14.S. Kruchinin, Rev. Theor. Sci. 4 (2016) 117-144.

15.S. Kruchinin, H. Nagao, S. Aono: Modern aspects of superconductivity: theory of superconductivity, World Scientific Pub. (2010) p.220. 